\title{
Effect of Ausforming on Retained Austenite After Continuous Cooling Transformation in a Medium-Carbon High Strength Steel
}

\author{
Man Liu ${ }^{a}$, Guang Xü ${ }^{a, *}$, Xin Chen ${ }^{a}$,Zhenye Chen ${ }^{b}$,Ziliu Xiong ${ }^{b}$ \\ ${ }^{a}$ Wuhan University of Science and Technology, The State Key Laboratory of Refractories and \\ Metallurgy, Wuhan 430081, China. \\ ${ }^{b}$ HBIS Group Technology Research Institute, Hebei Iron and Steel Group Co., Ltd., Shijiazhuang 05000, China.
}

Received: December 9, 2019; Revised: February 13, 2020; Accepted: March 6, 2020

\begin{abstract}
The effects of deformation on transformation kinetics during continuous cooling and microstructure evolution of a medium carbon high strength steel were investigated by metallographic method, dilatometry and X-ray diffraction etc. The results show that ausforming accelerated the ferrite and martensite transformation, but significantly retarded the bainite transformation. In addition, the amount of retained austenite decreased first and then increased, while the carbon content in retained austenite increased with the accumulation of strain. The change trend of the average dislocation density in martensite with strain showed the opposite trend as that of the amount of retained austenite. Moreover, the martensite laths were refined by ausforming. The work provides the theoretical reference for the design of parameters of thermalmechanical controlled process (TMCP) in the production of medium-carbon high strength steels.
\end{abstract}

Keywords: Ausforming, retained austenite, transformation, microstructure, dislocation density.

\section{Introduction}

Controlled rolling and controlled cooling technology, normally termed as thermalmechanical controlled process (TMCP), has been successfully adopted in the production of high strength steels for many years ${ }^{1,2}$. The continuous cooling process is normally used in the processing of steels. Different continuous cooling schedules lead to different microstructures and mechanical properties. Thus, the study on continuous cooling process of steels is important. Regarding to the high strength steels during continuous cooling, many studies were conducted on the continuous cooling transformation (CCT) curves $^{3-5}$. In addition, some investigations focused on effects of cooling rate and strain on microstructure evolution during continuous cooling ${ }^{6,7}$. Retained austenite (RA) and its stability play an important role in the mechanical properties of many steels, i.e. TRIP and Q\&P steels ${ }^{8,9}$. Author's previous work found that the carbon-enriched RA contributes to the elongation ${ }^{10}$. RA in Nb-bearing steel exhibits high stability due to its high stacking fault energy, which is caused by the dissolution of $\mathrm{Nb}$ in the final multi-phase microstructure ${ }^{11}$. Timokhina et al. ${ }^{12}$ reported that the stability of RA depends on the morphology and distribution of RA in microstructure.

Summarizing existing literatures, the stability of RA, which is affected by chemical composition, morphology and phase transition temperatures, is widely investigated. $\mathrm{Hu}$ et al. ${ }^{13}$ studied the effect of ausforming at bainite transformation temperature on RA in a C-Mn-Si bainitic steel. They claimed that the carbon content in RA increased first and then decreased with strain. However, few studies on the effect of high-temperature ausforming on RA with

*e-mail: xuguang@wust.edu.cn continuous cooling are reported. In addition, during the whole cooling process from austenitizing temperature, the diffusive transformation (ferrite transformation and bainite transformation) happens firstly at higher temperature, which is accompanied with carbon diffusion, and then the subsequent displacive transformation (martensite transformation) occurs without carbon diffusion. The larger the ausforming strain is, the more the dislocations and subgrains in the microstructure are induced. And the subsequent phase transformation is promoted by more nucleation sites provided by dislocations and subgrains, but the growth of grains is also retarded by dislocation and grain boundaries. Thus, the effect of ausforming on microstructure evolution during continuous cooling process and the RA at ambient temperature needs to be further clarified. Therefore, in the present study, the microstructure evolution during cooling process and relationship between ausforming and RA after continuous cooling were investigated. The purpose is to provide the theoretical reference for the design of processing parameters of TMCP in the actual production of medium-carbon high strength steels.

\section{Material and Experimental Procedures}

The tested steel with the chemical component of Fe-0.45C-2.03Si-2.81Mn (wt.\%) was refined in a laboratory-scale vacuum furnace of $50 \mathrm{~kg}$. The addition of silicon was to prevent the formation of cementite ${ }^{14,15}$. The ingots were first hot-rolled and then air-cooled to ambient temperature. Specimens were machined to $\varphi 6.0 \mathrm{~mm} \times 10.0 \mathrm{~mm}$ size for thermomechanical simulation tests. According to CCT curve of the tested steel, calculated and plotted by JMatPro 7.0 software 
in Figure $1^{16}$, the cooling speed of $1{ }^{\circ} \mathrm{C} / \mathrm{s}$ was adopted to obtain multi-phase microstructure. Gleeble 3500 simulator was used for thermal simulation experiments according to the procedures in Figure 2. Specimens were heated to $860^{\circ} \mathrm{C}$ for $15 \mathrm{~min}$ at a heating rate of $10^{\circ} \mathrm{C} / \mathrm{s}$ for austenitization. Then, the specimens were deformed with $0,25 \%$ and $50 \%$ strains at a strain rate of $1 \mathrm{~s}^{-1}$, respectively. Finally, all specimens were cooled at $1{ }^{\circ} \mathrm{C} / \mathrm{s}$ to ambient temperature. Here, the strain of $25 \%$ and $50 \%$ means true strain.

All specimens were prepared by mechanical polishing and etching for the subsequent microstructure observation. The mechanical polishing consisted of first grinding the specimens with a series of $\mathrm{SiC}$ papers of decreasing granularity and then polishing with diamond paste. The specimens were finally etched with $4 \%$ nital for a few seconds to reveal the microstructure. The examination of microstructure was conducted on a Nova 400 Nano field emission scanning electron microscope (SEM) and the acceleration voltage was set at $20 \mathrm{kV}$. Bruker diffractometer with $\mathrm{Co} \mathrm{K} \alpha$ radiation was selected for X-ray diffraction (XRD) experiments to measure the volume fraction of RA and the following equation was used to calculate the amount of austenite ${ }^{17}$. Three diffraction austenite peaks: $(200) \gamma,(220) \gamma$ and (311) $\gamma$ and two ferrite peaks: (200) $\alpha,(211) \alpha$ are selected for quantitative analysis.

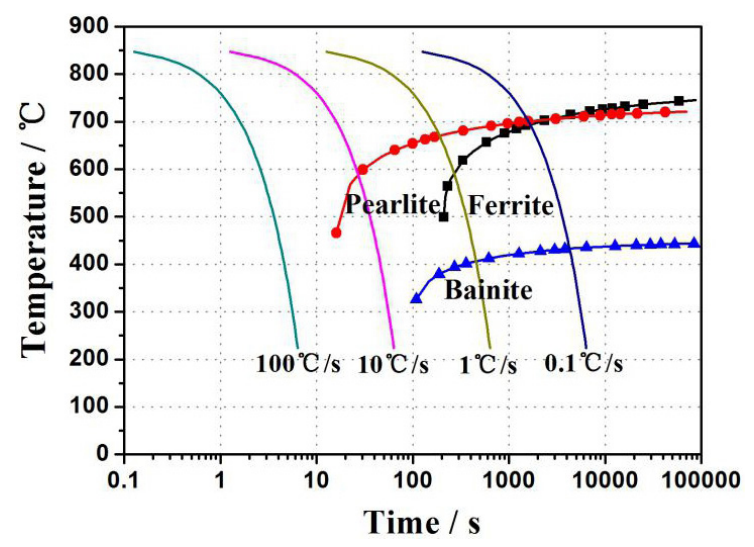

Figure 1. The CCT curve of the tested steel

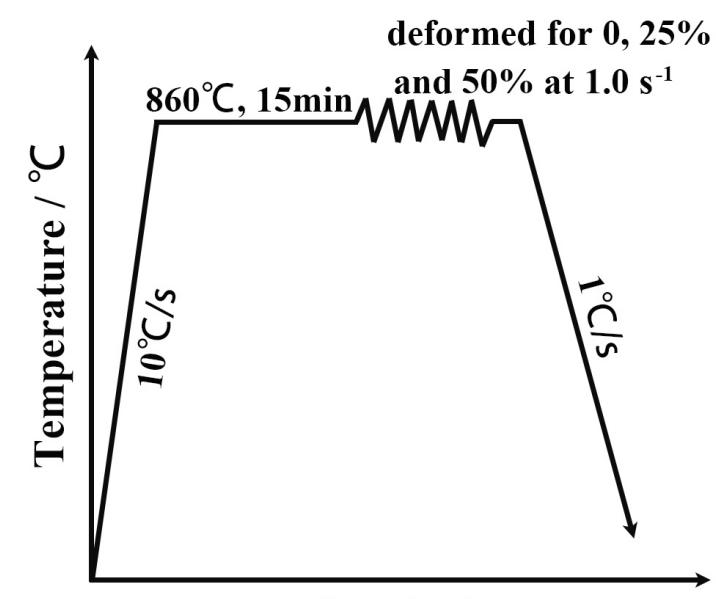

Time / min

Figure 2. The illustration of the experimental procedures

$$
V_{i}=\frac{1}{1+G\left(I_{\alpha} / I_{\gamma}\right)}
$$

where $V_{i}$ means the RA amount for each peak, $I_{\gamma}$ and $I_{\alpha}$ represent the integrated intensities of austenite and ferrite peaks, respectively. The following $G$ values for each peak are obtained from the reference ${ }^{17}: 2.46,1.32$ and 1.78 for $\mathrm{I}_{\alpha(200)} / \mathrm{I}_{\gamma(200)}, \mathrm{I}_{\alpha(200)} / \mathrm{I}_{\gamma(220)}$ and $\mathrm{I}_{\alpha(200)} / \mathrm{I}_{\gamma(311)}$, respectively, 1.21, 0.65 and 0.87 for $\mathrm{I}_{\alpha(211)} / \mathrm{I}_{\gamma(200)}, \mathrm{I}_{\alpha(211)} / \mathrm{I}_{\gamma(220)}$ and $I_{\alpha(211)} / I_{\gamma(311)}$, respectively. Carbon content in RA was calculated using formula $(2)^{18}$ :

$$
X_{C}=\frac{a-0.28664}{0.00028}
$$

where $\mathrm{a}$ is the lattice parameter of RA, $\mathrm{X}_{\mathrm{C}}$ is the carbon content in RA.

\section{Results and Discussion}

\subsection{Microstructural observation}

Figure 3 indicates the typical SEM microstructures of specimens deformed with different strains. According to the classification method to identify the microstructure proposed in Wang et al. ${ }^{19}$, it is observed that the microstructure of non-deformed specimen consists of ferrite (F), bainite (B), martensite $(\mathrm{M})$ or martensite/austenite $(\mathrm{M} / \mathrm{A})$ islands and RA. Ferrite is soft phase and is easy to be etched, showing in concave and polygonal morphology in the microstructure as pointed by arrows in Figure 3. Moreover, bainite phase presents the regular sheaves and contains interlath RA (shown in white film morphology) in SEM microstructure. Due to the addition of high silicon content, the precipitation of cementite was suppressed, resulting in the suppression of perilite. Thus, the stepwise decomposition of austenite into ferrite, bainite and martensite during continuous cooling process was observed at cooling speeds of $1{ }^{\circ} \mathrm{C} / \mathrm{s}$, which is consistent with the CCT curve in Figure 1. On the other hand, only polygonal ferrite and martensite form during continuous cooling process in deformed specimens, so the microstructure is composed of ferrite, martensite and RA. Compared to non-deformed specimen, the deformed specimens contain more ferrite and martensite laths are finer. No bainite is observed. This may be attributed to the fact that more nucleation sites are induced by deformation, which is beneficial to the ferrite transformation at high temperature, resulting in more ferrite in deformed specimen. In addition, the decomposition of austenite into ferrite is accompanied with carbon diffusion, thus the surrounding residual austenite is carbon-riched, causing higher chemical stability of residual austenite. Hence, the subsequent banite transformation is suppressed because of the high stability of residual austenite. Moreover, when the temperature is below martensite start temperature (Ms), the martensite starts to form. The growth of martensite is interfered by more grain boundaries or dislocations in deformed specimens, resulting in finer martensite laths, as shown in the enlarged images in Figure 3. Besides, some austenite is retained at ambient temperature. Furthermore, the amount of ferrite transformation increases and the martensite lath is further 
finer with strain. The more serious the deformation is, the more nucleation sites for ferrite transformation are induced, resulting in more ferrite. On the other hand, smaller grain size of austenite caused by severer deformation leads to the formation of finer martensite laths.
As an example to calculate the ferrite and martensite amounts, the micrographs of specimen with $25 \%$ strain are given in Figure 4. The darker areas contain ferrite and martensite (Figure 4a). Firstly, the darker areas are automatically colored in red by Image-Pro Plus software, as shown in
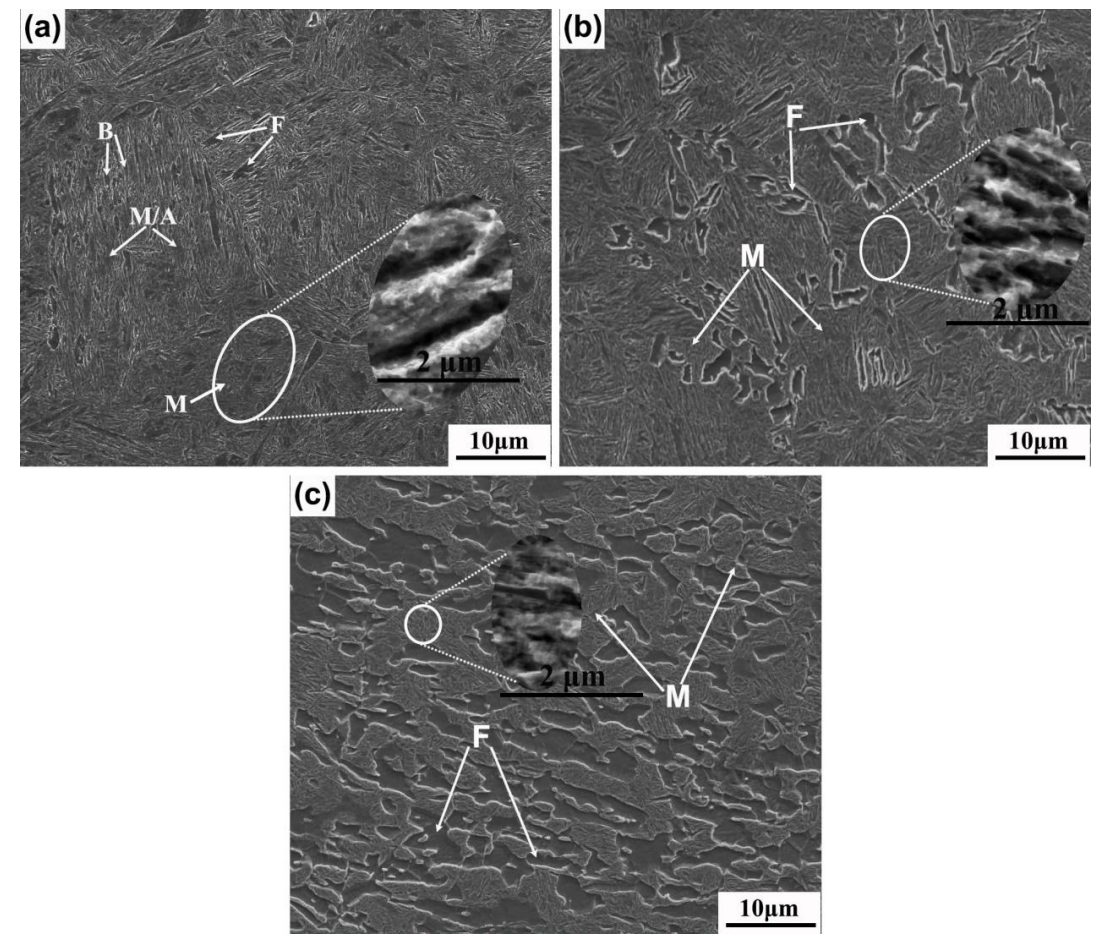

Figure 3. SEM microstructures of different specimens: (a) without deformation; (b) $25 \%$ strain; (c) $50 \%$ strain
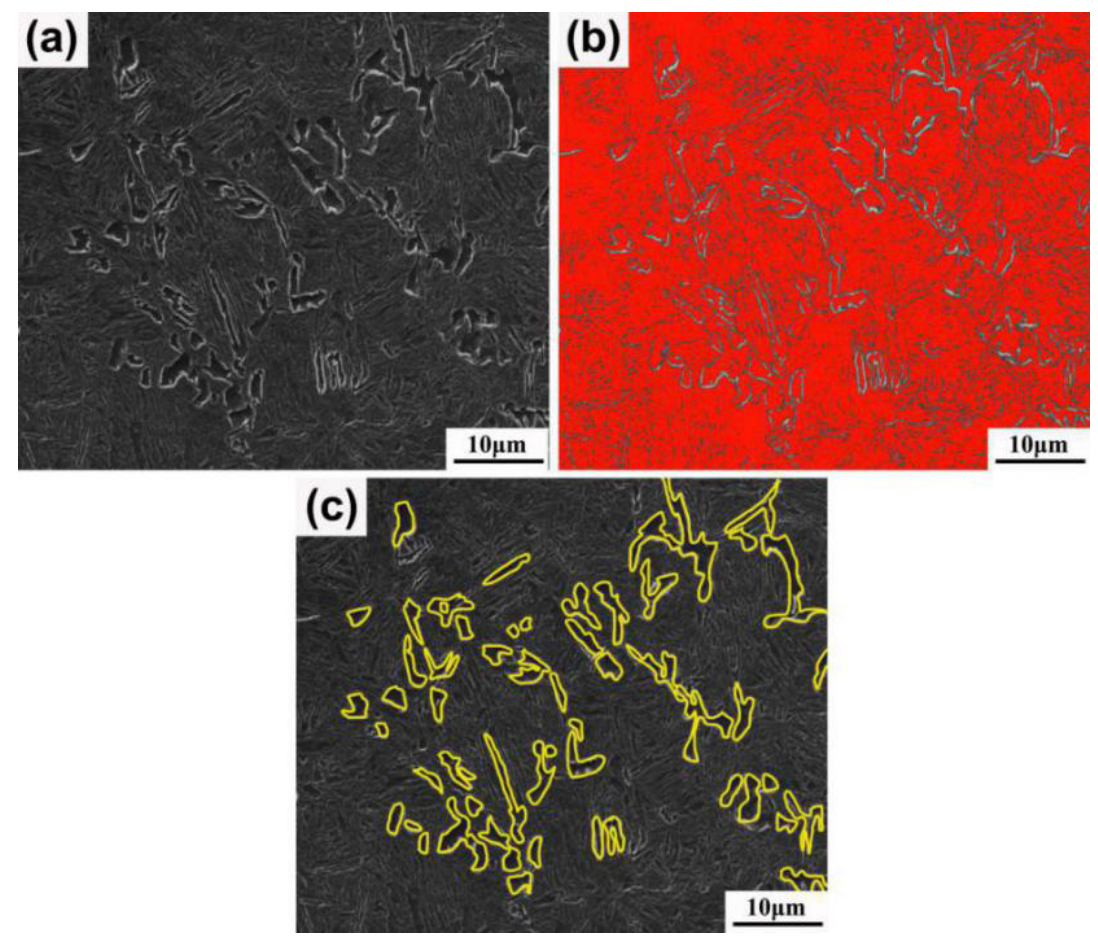

Figure 4. The example showing the method of calculating the volume fractions of ferrite and martensite: (a) the original micrograph; (b) the darker areas in Figure 4a are colored red; (c) the concave regions are manually marked. 
Figure $4 \mathrm{~b}$. The percentage of red areas is automatically calculated and the result is termed as $A_{1}$. Secondly, the concave areas belong to ferrite and it needs to be subtracted from red regions specially. The concave regions are carefully and manually marked (Figure 4c). The area percentage of concave areas (termed as $\mathrm{A}_{2}$ ) is measured by Image-Pro Plus software. Thirdly, the remaining part (termed as $\mathrm{A}_{3}$ ) is calculated by $A_{3}=A_{1}-A_{2}$, which should be the martensite amount. In this way, $\mathrm{A}_{1}$ and $\mathrm{A}_{2}$ are measured to be $87.48 \%$ and $17.35 \%$, respectively, so that $\mathrm{A}_{3}$ is $65.63 \%$. Therefore, the martensite and ferrite amounts in specimen with $25 \%$ strain were measured to be $65.13 \pm 6.58 \%$ and $18.35 \pm 3.04 \%$, respectively. Similarly, the martensite and ferrite amounts in specimen with $50 \%$ strain were determined to be $38.75 \pm 4.53 \%$ and $40.36 \pm 5.23 \%$, respectively. In addition, several typical SEM micrographs of each specimen were calculated for better accuracy of statistical results, and the average values are obtained as the final results.

\subsection{Analysis of dilation curves}

Figure 5 illustrates the dilatation curves of specimens under different ausforming conditions. The dilatation curves are the results of volume shrinkage caused by cooling and dilation caused by transformation. The amount of dilation is a function of temperature. No obvious ferrite transformation is observed and the small bainite transformation is shown at $461{ }^{\circ} \mathrm{C}$ (Figure 5a). It should be pointed out that a little ferrite exists in microstructure (Figure $3 \mathrm{a}$ ). This is not contradictory because only a certain amount of ferrite transformation can be shown in dilation curve during continuous cooling process. A sharp increase in dilation from point A to B in Figure 5b is caused by plastic deformation. It is noted that two visible slope changes in dilation curves are observed in deformed specimens (Figure 5b and 5c), indicating the occurrence of corresponding ferrite and martensite transformation. The ferrite transformation in deformed specimens demonstrates deformation induced ferrite transformation (DIFT). The DIFT is affected by strain amount. The larger the strain is, the earlier the DIFT occurs. Ferrite normally nucleates at the grain boundaries of austenite and defects. The austenite grains are broken and more dislocations are formed by ausforming ${ }^{20}$, resulting in the increase in the nucleation rate per unit volume of ferrite. On the other hand, more dislocations induced by ausforming are conducive to the diffusion of carbon atoms, resulting in shortening of inoculation period of ferrite transformation ${ }^{21}$. Therefore, the ferrite transformation is accelerated by ausforming. And the ferrite transformation happens earlier in $50 \%$ deformed specimen due to the severer deformation.

In addition, it is observed that the matensite start temperature (Ms) increases in deformed specimens. Due to the decomposition of austenite into ferrite, the ferrite/austenite $(\alpha / \gamma)$ interface is introduced in the microstructure, which provides more nucleation sites ${ }^{22}$. On the other hand, carbon is diffused into the surrounding austenite during ferrite reaction and the diffusion of carbon at the $\alpha / \gamma$ interfaces is much faster than that in austenite grains ${ }^{23}$. Thus, the carbon concentration near $\alpha / \gamma$ interface is higher than that in the center of austenite grain, resulting in the formation of carbon concentration gradients. Martensite transformation prefers to initiate at defects and carbon-depleted regions ${ }^{23}$. Therefore, ferrite transformation induced by ausforming accelerates the martensite transformation and results in the increase of $\mathrm{Ms}$ in deformed specimens. Moreover, the residual austenite becomes less and carbon content is higher because of the
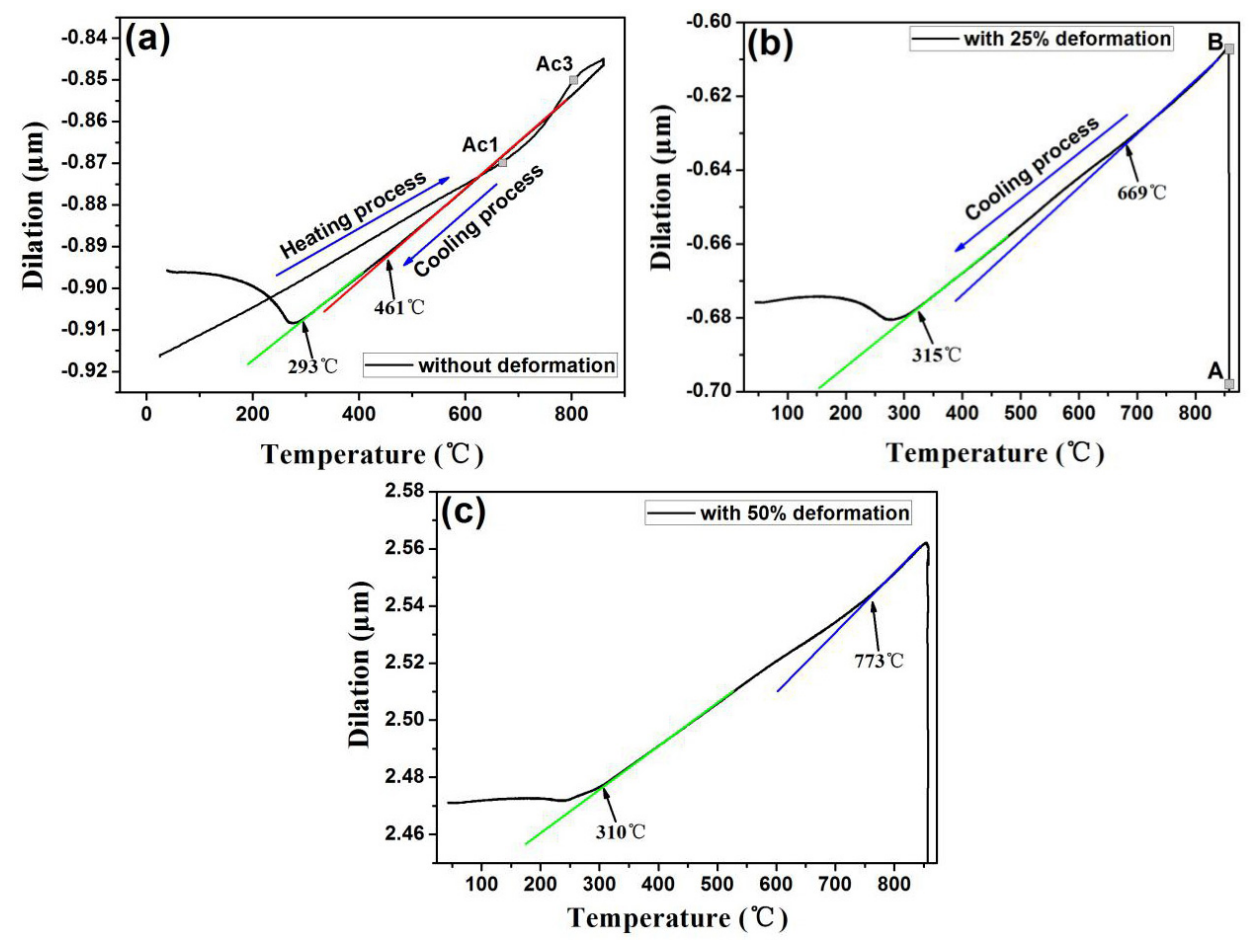

Figure 5. The dilation curves of different specimens: (a) without deformation; (b) $25 \%$ strain; (c) $50 \%$ strain 
Table 1. The volume fractions and carbon contents of RA and the average dislocation density in different specimens

\begin{tabular}{lccc}
\hline \multicolumn{1}{c}{ Specimens } & Without strain & With 25\% strain & With 50\% strain \\
\hline RA $(\%)$ & $19.91 \pm 3.13$ & $9.86 \pm 1.32$ & $19.88 \pm 3.08$ \\
\hline Carbon content (wt.\%) & $0.46 \pm 0.12$ & $0.54 \pm 0.18$ & $0.81 \pm 0.26$ \\
\hline Average dislocation density $\left(10^{13} / \mathrm{m}^{2}\right)$ & 3.65 & 8.97 & 3.99 \\
\hline
\end{tabular}
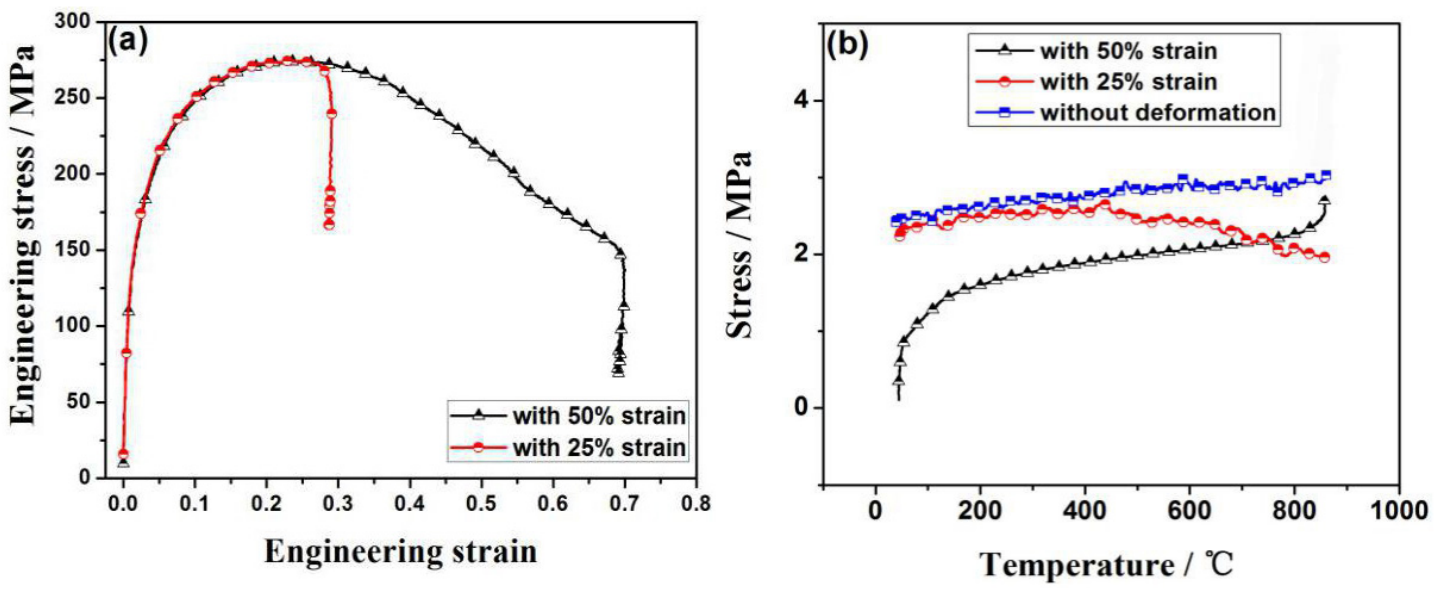

Figure 6. (a) Stress-strain curves with different strains during deformation; (b) stress state during cooling period versus temperature

rejection of carbon from ferrite in specimen with 50\% strain compared to that in specimen with $25 \%$ strain. Hence, higher chemical stability of austenite due to higher carbon content plays an important role in subsequent martensite transformation, resulting in lower $\mathrm{Ms}$ in specimen with $50 \%$ strain compared to that in specimen with $25 \%$ strain.

Figure 6 a gives the stress-strain curves of deformed specimens. The flow stress increases first and then decreases with the accumulation of strain. The maximum flow stress is the same for deformed specimens. It indicates that the maximum flow stress is independent of deformation strain because the maximum flow stress occurs at about $20 \%$ strain, while it is depended on the strain rate and deformation temperature ${ }^{24,25}$. In addition, the literature has proven that stress affects the transformation kinetics ${ }^{10}$. Hence, it is necessary to analyze the stress on specimens during continuous cooling. Figure $6 \mathrm{~b}$ shows the stress state during cooling process versus temperature, illustrating small stress less than $3 \mathrm{MPa}$ on the samples during continuous cooling process. A stress as small as possible was applied on samples during continuous cooling in order to fix the specimen in place. According to the result by Zhou et al. ${ }^{26}$, the transformation will not be affected by small stress less than $3 \mathrm{MPa}$. Therefore, the impact of stress on transformation can be ignored.

\section{$3.3 R A$ and carbon content}

Figure 7 illustrates the diffraction patterns of undeformed and deformed specimens by XRD experiments. The angles and integrated intensities of diffraction peaks were decided by HighScore Plus software. According to Equations 1 and 2, the volume fractions and carbon concentrations in RA were calculated and the average values of $\mathrm{V}_{\mathrm{i}}$ and carbon content were gained as the final results and the corresponding results are given in Table 1 . It exhibits that the volume fraction of RA decreases first and then increases, while the

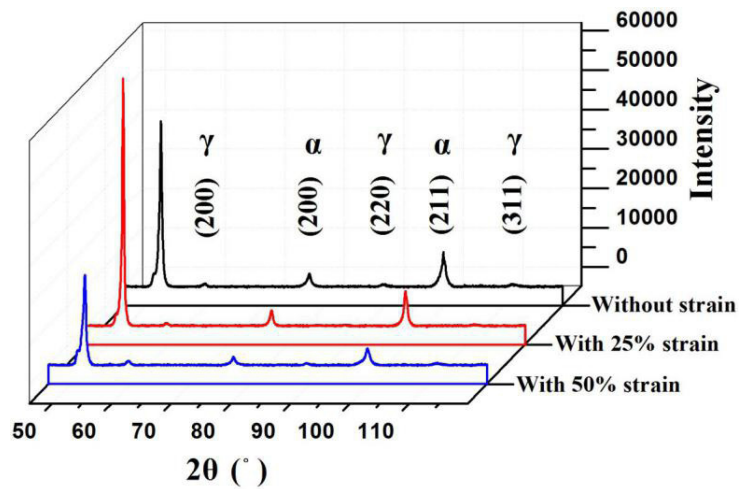

Figure 7. The diffraction peaks of specimens

carbon content increases with strain. This is because only a few bainite and ferrite form during continuous cooling in non-deformed specimen, resulting in less carbon diffusing into the surrounding austenite, thus the carbon content of RA is lower in specimen without deformation. On the other hand, more ferrite forms by ausforming and more carbon atoms are rejected to adjacent austenite. Hence, the carbon content is higher in deformed specimen. In addition, more ferrite in specimen with $25 \%$ strain means less residual austenite for subsequent transformation. Thus, the residual austenite amount decreases after marteniste transformation, leading to less RA at ambient temperature compared to the non-deformed specimen. Besides, the ferrite transformation is further promoted in specimen with $50 \%$ strain by severer deformation, resulting in the higher carbon content in residual austenite. Hence, the following martensite transformation is retarded due to the higher stability of austenite, leading to more RA at ambient temperature compared to that in specimen with $25 \%$ strain. 


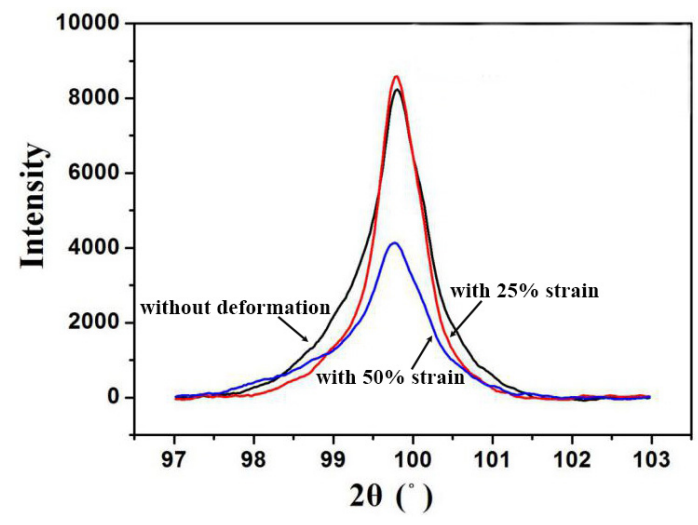

Figure 8. The (211) $\alpha$ diffraction peaks in different specimens

The average dislocation density (ADD) in ferrite can be measured by XRD experiments 27,28 . The values of ADD in different specimens were calculated by Equation 3 proposed by Dunn and $\operatorname{Kogh}^{29}$.

$$
\rho=\beta^{2} /\left(4.35 \times b^{2}\right)
$$

where $\rho$ represents the ADD in ferrite, $\beta$ means the half-width of (211) $\alpha$ and $b$ is Burgers vector of $0.248 \mathrm{~nm}$.

Figure 8 shows the (211) $\alpha$ diffraction peaks of different specimens and the calculated results of the ADD are given in Table 1. It is observed that the ADD increases first and then decreases with strain. Compared with the dislocation density in non-deformed specimen, more dislocations are generated by ausforming, thus the average dislocation density obviously increases in deformed specimens with $25 \%$ strain. Moreover, the average dislocation density decreases when the strain increases to be $50 \%$. This is because that dynamic recrystallization happens during deformation process, which is proved in Figure 6a. The flow stress reaches the maximum value when the strain is about $20 \%$ and after that, the stress is decreased with strain, which means the occurrence of dynamic recrystallization and results in the reduction of dislocation density. Therefore, the average dislocation density is decreased in the specimen with $50 \%$ strain.

\section{Conclusions}

The effects of deformation on transformation kinetics during continuous cooling and microstructure evolution of a medium carbon high strength steel were investigated by metallographic method, dilatometry and XRD experiments. The results show that ausforming promoted ferrite transformation by increasing the nucleation rate per unit volume and shortening of inoculation period of ferrite transformation. The larger the strain was, the greater the promotion degree was. In addition, the subsequent decomposition of austenite into bainite was hindered by ferrite transformation. Moreover, the Ms increased in deformed specimens by more martensite embryos at the $\alpha / \gamma$ interfaces, and the martensite laths was refined because the growth was retarded by more grain boundaries and dislocations in deformed specimens. Besides, the volume fraction of RA decreased first and then increased, while the carbon content in RA increased with strain. This is because more ferrite formed in deformed specimens accompanied with more carbon atoms rejecting to the adjacent austenite, resulting in the increase of carbon content in RA. Finally, average dislocation density increased first and then decreased with the accumulation of strain.

\section{Acknowledgments}

The authors gratefully acknowledge the financial supports from the National Natural Science Foundation of China (NSFC) (No.51874216 and 51704217), the Major Project of Technology Innovation of Hubei Province (No.2017AAA116), the Special Project of Technological Innovation of Wuhan (2018010402011187) and the Hebei Joint Research Fund for Iron and Steel (E2018318013).

\section{References}

1. Schwinn V, Bauer J, Flüss P, Kirsch HJ, Amoris E. Recent developments and applications of TMCP steel plates. Rev Metall. 2011;108(5):283-94.

2. Yu C, Gao XH, Wang P, Niu XX, Qiu CL, Du LX, et al. Effect of TMCP parameters on microstructure and mechanical properties of cargo oil tanks steel. Appl Mech Mater. 2014;577:77-80.

3. Wang X, Kang Y, Yu H, Chen L, Kong Q. Dynamic CCT diagram of automobile beam steel with high strength produced by FTSR technology. J Iron Steel Res Int. 2008;15(2):60-4.

4. Xu FY, Wang YW, Bai BZ, Fang HS. CCT curves of low-carbon Mn-Si steels and development of water-cooled bainitic steels. J Iron Steel Res Int. 2010;17:46-50.

5. Falkenreck T, Kromm A, Böllinghaus T. Investigation of physically simulated weld HAZ and CCT diagram of HSLA armour steel. Weld. World. 2018; 62(1): 47-54.

6. Van Bohemen SMC, Sietsma J. The kinetics of bainite and martensite formation in steels during cooling. Mater Sci Eng A. 2010;527(24-25):6672-6.

7. Wang BX, Jiang YT, Xu XD, Liu XH, Wang GD. Effect of austenite deformation on continuous cooling transformation microstructures for $22 \mathrm{CrSH}$ gear steel. J Iron Steel Res Int. 2007;14(1):69-73.

8. Lee S-J, Lee S, De Cooman BC. Mn partitioning during the intercritical annealing of ultrafine-grained $6 \% \mathrm{Mn}$ transformationinduced plasticity steel. Scr Mater. 2011;64(7):649-52.

9. Shi J, Sun XJ, Wang MQ, Hui WJ, Dong H, Cao WQ. Enhanced work-hardening behavior and mechanical properties in ultrafinegrained steels with large-fractioned metastable austenite. Scr Mater. 2010;63(8):815-8.

10. Liu M, Xu G, Tian JY, Yuan Q, Zhou MX, Hu HJ. The effect of stress on bainite transformation, microstructure, and properties of a low-carbon bainitic steel. Steel Res Int. 2019;90(10):1900159. http://dx.doi.org/10.1002/srin.201900159.

11. Feng QX, Li LF, Yang WY, Sun ZQ. Effect of Nb on the stability of retained austenite in hot-rolled TRIP steels based on dynamic transformation. Mater Sci Eng A. 2014;603:169-75.

12. Timokhina IB, Hodgson PD, Pereloma EV. Effect of microstructure on the stability of retained austenite in transformation-inducedplasticity steels. Metall Mater Trans, A Phys Metall Mater Sci. 2004;35(8):2331-41.

13. Hu HJ, Xu G, Wang L, Zhou MX, Xue ZL. Effect of ausforming on the stability of retained austenite in a C-Mn-Si bainitic steel. Met Mater Int. 2015;21(5):929-35.

14. Chen GH, Xu G, Zurob HS, Hu HJ, Wan XL. Effect of strain rate on the bainitic transformation in Fe-C-Mn-Si medium-carbon bainitic steels. Metall Mater Trans, A Phys Metall Mater Sci. 2019;50(2):573-80. http://dx.doi.org/10.1007/s11661-018-5051-z. 
15. Liu M, Xu G, Tian JY, Wei ZR, Zhang QX. Effect of Si content on the kinetics of bainitic transformation and properties of low carbon bainite steels. J. Iron Steel Res. 2019;11:982-7.</jrn> (In Chinese)

16. Tian JY, Xu G, Zhou MX, Hu HJ. Refined bainite microstructure and mechanical properties of a high-strength low-carbon bainitic steel treated by austempering below and above $\mathrm{M}_{\mathrm{s}}$. Steel Res Int. 2018;89(4):1700469. http://dx.doi.org/10.1002/srin.201700469.

17. Singh SB, Bhadeshia HKDH. Estimation of bainite plate-thickness in low-alloy steels. Mater Sci Eng A. 1998;245(1):72-9. http:// dx.doi.org/10.1016/S0921-5093(97)00701-6.

18. Cheng L, Böttger A, De Keijser TH, Mittemeijer EJ. Lattice parameters of iron-carbon and iron-nitrogen martensites and austenites. Scr Mater. 1990;24(3):509-14.

19. Wang CY, Shi J, Cao WQ, Dong H. Characterization of microstructure obtained by quenching and partitioning process in low alloy martensitic steel. Mater Sci Eng A. 2010;527(15):3442-9.

20. Hu HJ, Xu G, Dai FQ, Tian JY, Chen GH. Critical ausforming temperature to promote isothermal bainitic transformation in prior-deformed austenite. Mater Sci Technol. 2019;35(4):420-8.

21. Yang ZM. Influence of deformation parameters on Deformation Induced Ferrite Transformation (DIFT) in plain low carbon steel. Mater Sci Forum. 2007;539:4704-7.

22. Girault E. Bainitic transformation in trip-assisted steels and its influence on mechanical properties. [thesis]. Belgium: Department of Metallurgy and Materials Engineering, K.U. Leuven; 1999.
23. Zhu KY, Chen H, Masse JP, Bouaziz O, Gachet G. The effect of prior ferrite formation on bainite and martensite transformation kinetics in advanced high-strength steels. Acta Mater. 2013;61(16):6025-36.

24. Chen GH, Xu G, Hu HJ, Yuan Q, Zhang QX. Effect of strain rate on deformation resistance during ausforming in Fe-C-Mn-Si high-strength bainite steels. Steel Res Int. 2018;89(11):1800201. http://dx.doi.org/10.1002/ srin.201800201.

25. Hu HJ, Xu G, Wang L, Zhou MX. Effects of strain and deformation temperature on bainitic transformation in a Fe-C-Mn-Si alloy. Steel Res Int. 2017;88(3):1600170. http://dx.doi.org/10.1002/ srin.201600170.

26. Zhou MX, Xu G, Wang L, Yuan Q. The varying effects of uniaxial compressive stress on the bainitic transformation under different austenitization temperatures. Metals. 2016;6(5):119.

27. Liang ZY, Li YZ, Huang MX. The respective hardening contributions of dislocations and twins to the flow stress of a twinning-induced plasticity steel. Scr Mater. 2016;112:28-31.

28. Li ZC, Ding H, Misra RDK, Cai ZH. Deformation behavior in cold-rolled medium-manganese TRIP steel and effect of prestrain on the Lüders bands. Mater Sci Eng A. 2017;679:230-9.

29. Dunn CG, Kogh EF. Comparison of dislocation densities of primary and secondary recrystallization grains of Si-Fe. Acta Mater. 1957;5(10):548-54. 